\title{
GENETIC ALGORITHM FOR SELF OCCLUSION GAIT MODEL
}

\author{
Precila Mary $\mathbf{R}^{1}$ Femila Goldy $\mathbf{R}^{2}$ \\ ${ }^{1}$ RMK Engineering College, Kavaraipettai, Gummidipoondi \\ ${ }^{2}$ Anand Institute of Higher Technology, Kazhipattur, Chennai. \\ Email: ${ }^{1}$ rpm.cse@rmkec.ac.in, ${ }^{2}$ femilajoyrobert@gmail.com
}

\begin{abstract}
Human identification has recently gained growing interest from computer vision researchers. Gait recognition domain has a wide range of applications in medicine (biomechanics), mathematics, psychology, rehabilitation and sports activity. It has the unique capability to recognize people at a distance when other biometrics is obscured. This paper aims to handle high levels of occlusion, which is of special importance in gait as the human body is self-occluding. Initially, the silhouettes segmented from video sequences are noisy and need further processing. Mathematical morphological operations are employed for holes remedy and noise elimination. To deal with the incomplete gait image, a new gait pattern representation method named frame difference energy image (FDEI) is used. After the foregoing preprocessing steps, then extract the self occluded feature from the silhouette image sequences. Then, warped template is updated by eliminating self-occluded part. Finally, the genetic algorithm is proposed for recognition of human walking.
\end{abstract}

Keywords: Gait Recognition, Self Occlusion, Frame Difference Energy Image, Genetic Algorithm.

\section{INTRODUCTION}

Human gait recognition as a new biometric aimed to recognize person via the style of people walking, which contains physiological or behavioral characteristics of human. The need for automated person identification is growing in many applications such as surveillance, access control and smart interfaces. It is well-known that biometrics are a powerful tool for reliable automated person identification. Established biometric-based identification techniques range from fingerprint and hand geometry methods to schemes like face recognition and iris identification. However, these methodologies are either intrusive or restricted to very controlled environments. For example, current face recognition technology is capable of recognizing only frontal or nearly frontal faces. When the problem of person identification is attempted in natural settings, such as those that occur in the automatic surveillance of people in strategic areas, it takes on a new dimension. Biometrics such as fingerprint or iris is then no longer applicable. Furthermore, night vision capability (an important component in surveillance) is usually not possible with this biometrics. A biometric that can address some of these shortcomings is the human 'gait'. The attraction of using gait as a biometric is that it is nonintrusive and typifies the motion characteristics specific to an individual. It is a well-known fact that people often recognize others by simply observing their gait which may justify using it as a cue for recognizing people.

\section{A. Gait Cycle}

A gait cycle is the time interval between successive instances of initial foot-to-floor contact 'heel strike' for the same foot. The cycle begins with the heel strike of one foot which marks the start of the stance phase. The ankle flexes to bring the foot flat on the floor and the body weight is transferred onto it. The other leg swings through in front as the heel lifts of the ground. As the body weight moves onto the other foot, the supporting knee flexes. The remainder of the foot, which is now behind lifts off the ground ending the stance phase. The start of the swing phase is when the toes of the foot leave the ground. The weight is transferred onto the other leg and the leg swings forward to meet the ground in front of the other foot. The gait cycle ends with the heel strike of the foot. Fig.1. shows the gait cycle.

\section{RELATED WORK}

Haiping $L u$ et al[1] proposed the feature extraction is determined by a multi linear projection that captures most of the original tensorial input variation.An MPCA based tensor object recognition framework is applied to the problem gait recognition.Nikolas V.Boulgouris et al[2] proposed the Radon Transform of binary silhouettes for gait representation and recognition. Dimosthenis loannidis et al[3] proposed an innovative gait identification and authentication method based on the use of novel 2-D and 3-D features. Furthermore, the use of a genetic algorithm is 


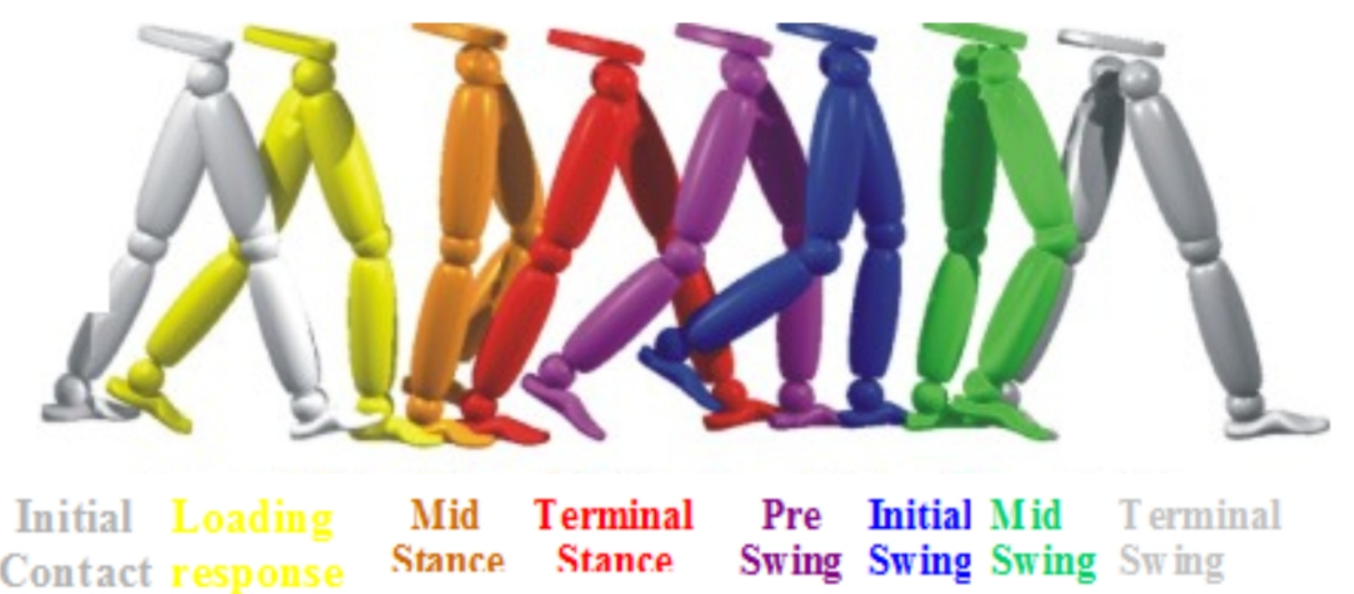

Fig. 1.Gait Cycle

presented for fusing information from different feature extractors. Dacheng Tao et al[4] proposed the developed gabor gait methods and GTDA methods are combined with LDA for gait recognition. Ju Han et al [5] proposed a new spatio temporal gait recognition called Gait Energy image, to characterize human walking properties for individual recognition by gait. Dong $\mathrm{Xu}$ et al,[6] explored gait recognition based on a matrix representation. a supervised algorithm namely discriminant analysis with tensor representation is applied to further improve classification strategy. Zingy Liu et al[7] presented a novel model based hidden markov model (HMM), coupled with an eigen-stance model, to correct for common errors in silhouette detection arising from shadows and background subtraction. Amit Kale et al,[8] proposed a view-based approach to recognize humans from their gait. To obtain the observation vector from the image features, they employ indirect and direct approach. Liang Wang et al,[9] proposed a simple and efficient automatic gait recognition algorithm using statistical shape analysis. Er-hu Zhang et al, [10] proposed a gait recognition method with Dynamic Gait Energy Image and Manifold Learning. Dynamic Gait Energy is used to extract the dynamic variance parts of the silhouettes of the motion body corresponding to the gait averaged image in a walking sequence.

\section{PROPOSED WORK}

The proposed system is to recognize the way of walking using Genetic algorithm. Initially, the silhouettes segmented from video sequences are noisy and need further processing. Mathemetical morphological operations are employed for holes remedy and noise elimination.To deal with the incomplete gait image,a new gait pattern representation method named Frame Difference Energy Image(FDEI) is used.After the foregoing preprocessing steps, the self occluded features are extracted from the silhouette image sequences. Finally, Fig. 2. shows the block diagram of the proposed methodology

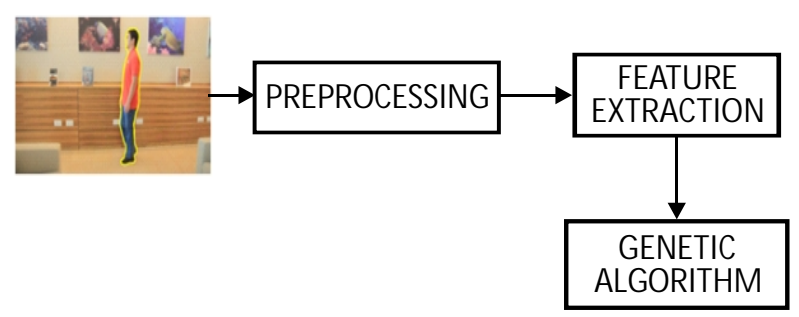

Fig. 2. Functional Block Diagram

\section{A. Preprocessing}

\section{Silhouette Extraction}

The silhouette is usually extracted by finding the difference between the background and current image or grouping optic flow to find the coherent motion.

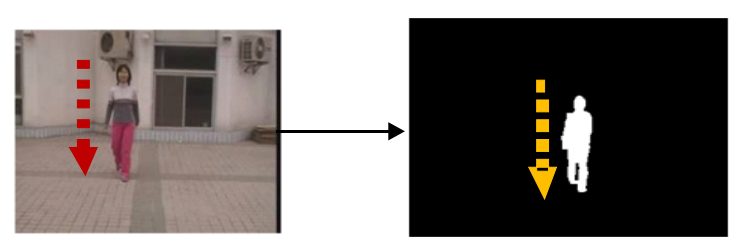




\section{Noise Removal}

There are inevitably spurious pixels, holes inside moving subject and other anomalies in the detected sections. Mathematical morphological operations, such as erosion and dilation, are widely used to remove spurious pixels and fill small holes inside the extracted silhouettes.

\section{Incomplete silhouette}

Gait recognition depends on two types of information:

- Shape information (static information).

- Dynamic information.

Shape information refers to the appearance, such as, body height, width, body-part proportions, hair, hunching, dressing, taking a package or ball and so on. Some shape information remains unchangeable or similar during people walking. Information extracted from a single frame belongs to shape information.Dynamic information characterizes the motion changes of the human body regardless of the underlying structure such as,

- Joint angles trajectories of the lower limbs.

- Shape of motion features obtained by optical flow.

Fig. 3. shows the example of incomplete sihouette
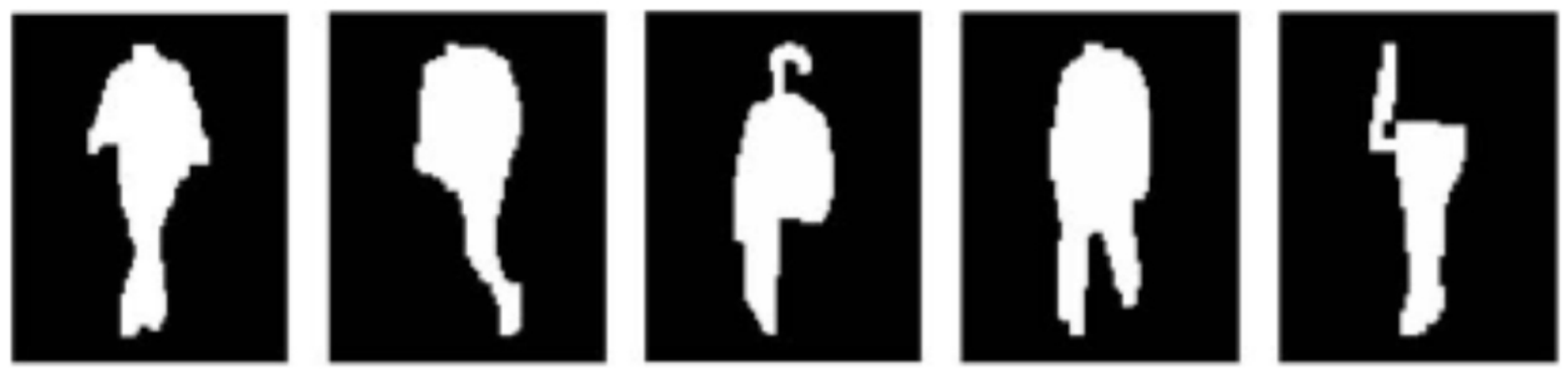

Fig. 3. Examples of incomplete silhouettes
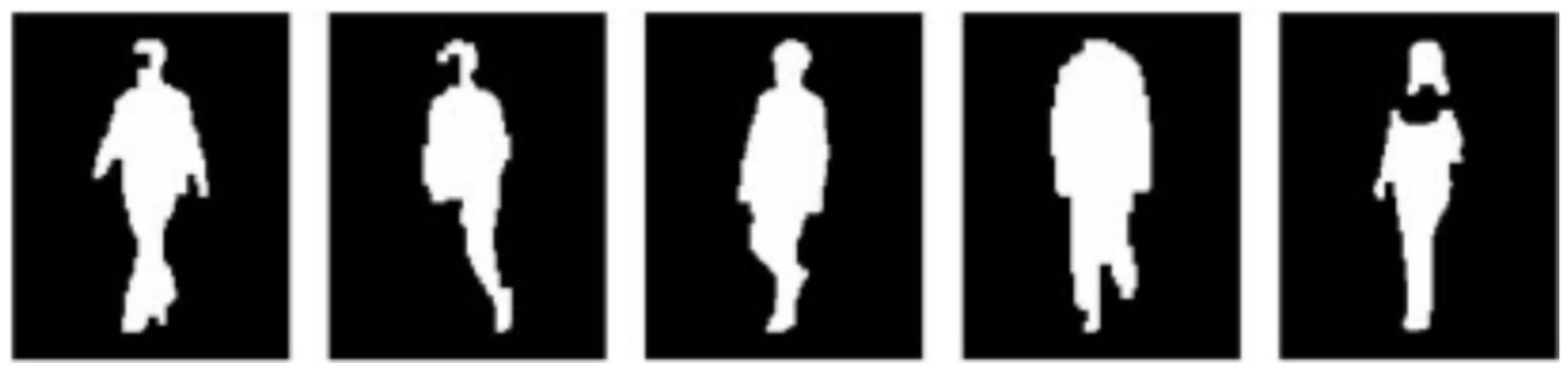

Fig. 4. Foregoing frames corresponding to the silhouettes in the first row
Gait Energy Image is an effective representation scheme with good discriminating power and robustness against segmental errors. It saves both storage space and computation time for recognition and is less sensitive to silhouette noise in individual frames. GEI is defined as follows:

$G(x, y)=\frac{1}{N} \sum_{t=1}^{N} B(x, y, t)$

$N$ - number of frames per cycle.

$t$ - sequence number of the frame in the cycle

$B(x, y, t)$ - silhouette image at time $t$.

$G(x, y)-$ Gait energy image.

Example is illustrated in fig. 4.

\section{STEP 2: Constructing FDEI}

The FDEI of $B(x, y, t)$ can be represented as follows:

$B(x, y,(t-1))$ - Last frame of the cycle.

$B(x, y, t)$ - silhouette image at time $t$.

Example is illustrated in fig. 5. 

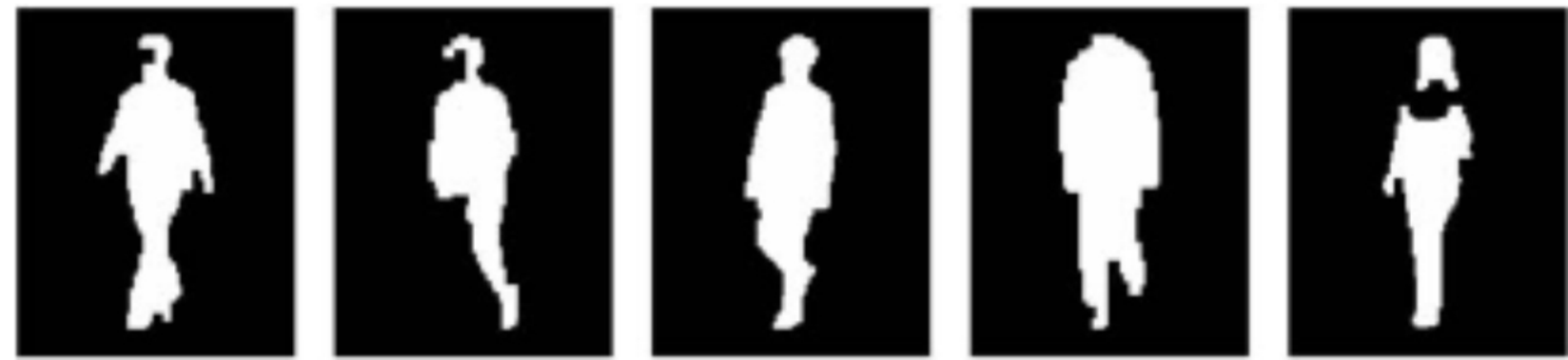

Fig. 5. FDEI of the silhouettes in the first row.

\section{B. Feature Extraction}

After the foregoing processing steps, then extract the self occluded features from the silhouette image sequences.

\section{Self Occlusion Feature}

In general, determining an occlusion or self-occlusion of the object at time $t$ cannot be done from the image $I_{t}$ alone without prior knowledge of the $3 \mathrm{D}$ scene, and in a general tracking scenario, 3D scene information is unavailable. However, the occlusion (self and disocclusions) can be partially resolved by observing multiple images from differing viewpoint in a process of movement of the observer as shown in fig. 6 . In the specific case of a moving and deforming object, given an estimate of the object projected into the imaging plane at time $t$ and the image at time $t+1$, this observation can be readily applied to determine the occluded region $\mathrm{O}_{t}$. In fact, the principle is that the occluded region $O_{t}$ is the subset of $R_{t}$ that does not correspond to a region in $I_{t+1}$.

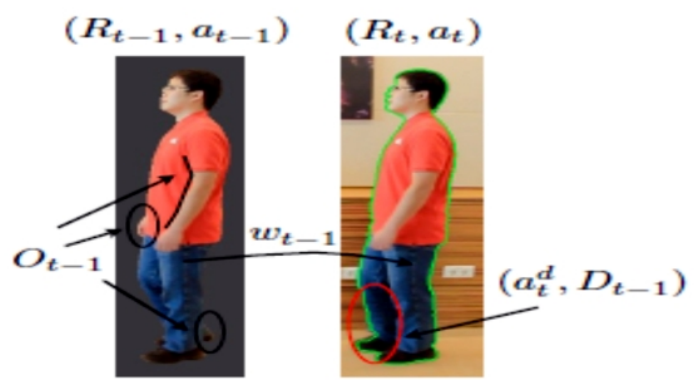

Fig. 6. Self Occlusion Representations

The self-occlusions Ot-1 are indicated in black, self-disocclusions Dt-1 are in red.

This includes both occlusions and selfocclusions. The case of disocclusions and self-disocclusions of the object cannot be resolved given $R_{t}$ and $I_{t+1}$ without a prior notion of the object. Indeed, if a part of the object projected in the imaging plane comes in view at time $t+1$ and it was not observed earlier, then it is impossible to know whether the part newly in view is part of the object of interest, another object or the background without additional assumptions on the object.

\section{Steps in Self Occlusion Gait Model:}

1. The object is segmented from the starting frame (that is all the training required), and determines precise shape of object in all subsequent frames i.e, from current template to object in next frame (self-occlusions are simultaneously detected) and the correspondence is valid under large deformation and motion as shown in fig. 7 .

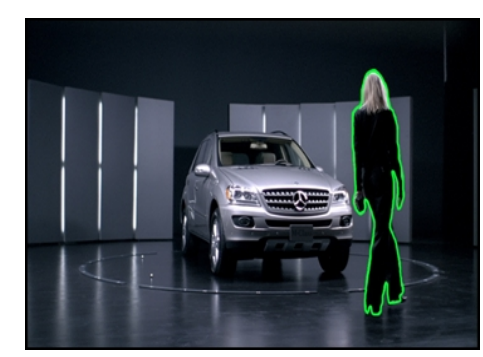

Frame 1

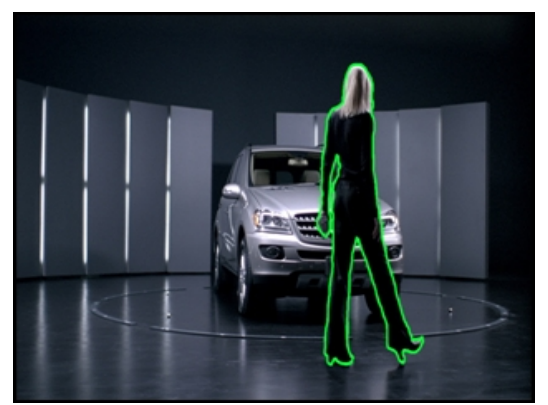

Frame 2 (Self occluded)

Fig. 7. Example of Self occlusion Gait 
2. Warped template is updated by eliminating self-occluded part.

3. Self-disocclusions (the part of the object that comes into view at the current frame) is detected and the template is updated

4. The change of appearance is taken into account and the template is updated by robust appearance filtering via a non-linear observer

5. Steps $2-5$ are repeated with the newly updated template and the next frame.

\section{Gait Recognition}

Recognition of human walking is done by genetic algorithm. The data processed by the GA are a set (population) of strings. Each string has its own fitness value determined by an objective function value of the corresponding point in a search space. Based on fitness values, the GA decides the probability of each string in the current generation to be processed by genetic operators and produces offspring. There are three genetic operators: selection, crossover and mutation. A selection operator chooses strings to undergo crossover and mutation (so-called selection for reproduction), and strings to constitute the next generation among the generated offspring and the current strings (so-called selection for replacement), based on their fitness values. With probability $p c$, two selected strings are crossed over at the crossover site, which is randomly chosen. The role of crossover is to generate better solutions by exchanging effective information contained in good solutions. Mutation changes the value of the randomly chosen site from 0 to 1 with probability $p m$. By introducing extra variation to the population, it allows the genetic search to continue without losing diversity. These three operators are used successively to generate the next generation. The evolutionary cycle of selection and genetic perturbation is repeated until the termination condition is satisfied.

\section{CONCLUSION}

With the increasing demands of visual surveillance systems human identification at a distance has recently gained more interest. Gait is a potential behavioral feature and many allied studies have demonstrated that it can be used as a biometric feature for recognition. The development of computer vision techniques has also assured that vision based automatic gait analysis can be gradually achieved. The proposed system is used to recognize the way of walking in real time environment using genetic algorithm.

\section{REFERENCES}

[1] Haiping Lu, Konstantinos N.Plataniotis, Anastasios N.Venetsanopoulos"Multilinear Principal Component Analysis Of Tensor Objects", IEEE Transactions on Neural Networks, Vol 19, No 1, January 2008.

[2] Nikolas V.Boulgouris and Zhiwei X.Chi "Gait Recognition Using Radon Transform And Linear Discriminant",IEEE Transactions on Image Processing,Vol.16,No.3,March 2007.

[3] Dimosthenis loannidis, Dimitrios Tzovaras, Ioannis G.Damousis, Savvas Argyropoulos and Konstantinos Moustakas "Gait Recognition Using Compact Features Extraction Transforms And Depth Information" IEEE Transactions on Information Forensics and security, vol.2, No 3, September 2007.

[4] Dacheng Tao,Xuelong $\mathrm{Li}$, Xindong $\mathrm{Wu}$,Stephen J.Maybank "General Tensordiscriminant Analysis And Gabor Features For Gait Recognition", IEEE Transactions on Pattern Analysis and Machine Intelligence.

[5] Ju Han, Bir Bhanu "Individual Recognition Using Gait Energy Image" IEEE Transactions on pattern Analysis and Machine Intelligence, Vol 28, No. 2, February 2006.

[6] Dong $X u$,Shuicheng Yan,Dacheng Tao,Lei Zhang,Xuelong Li and Hong-Jiang Zhang "Human Gait Recognition With Matrix Representation" IEEE Transactions on Circuit and systems for video technology, July 2006

[7] Zingy Liu and Suede Sarkar "Effect Of Silhouette Quality On Hard Problems In Gait Recognition" IEEE Transaction on Systems, Man and Cybernetics, Vol 35 No.2 April 2005.

[8] Amit Kale, Aravind Sundaresan, A. N. Rajagopalan, Naresh P. Cuntoor, Amit K. Roy-Chowdhury, Volker Krüger and Rama Chellappa "Identification Of Humans Using Gait" IEEE Transactions On Image Processing Vol. 13, No. 9, September 2004.

[9] Liang Wang, Tieniu Tan, Weiming Hu, Huazhong Ning "Automatic Gait Recognition Based On Statistical Shape Analysis" IEEE Transactions on image processing, Vol 12, No 9, September 2003.

[10] Er-hu Zhang,Hua-Bing Ma,Ji-Wen Lu,Ya-Jun Chen "gait Recognition Using Dynamic Gait Energyand PCA+LPP Method" Proceedings of the Eight International Conference on Machine Learning and Cybernatics,July 2009. 


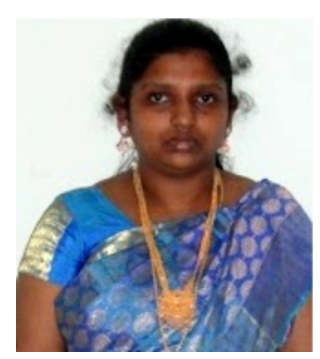

Precila Mary.R, Assistant Professor, Department of Computer Science and Engineering, R.M.K Engineering College, Kaveraipettai. She has presented several papers in National and International Conferences.

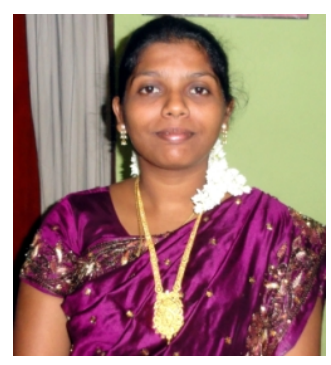

Femila Goldy.R, Lecturer Department of Computer Science and Engineering, Anand Institute Of Higher Technology, Chennai.Her area of interest includes Image Processing, Biometrics and Visual Surveillance. She has presented several papers in National and International Conferences. 